\title{
Identification of target genes of PAX3-FOXO1 in alveolar rhabdomyosarcoma
}

\author{
EUN HYUN AHN ${ }^{1,2}$, GABRIELA E. MERCADO ${ }^{1,5}$, MARICK LAÉ $^{3,6}$ and MARC LADANYI ${ }^{3,4}$ \\ ${ }^{1}$ Department of Pathology and Laboratory Medicine, University of Pennsylvania School of Medicine, Philadelphia, PA 19104; \\ ${ }^{2}$ Department of Pathology, University of Washington School of Medicine, Seattle, WA 98195; ${ }^{3}$ Department of Pathology \\ and ${ }^{4}$ Human Oncology and Pathogenesis Program, Memorial Sloan-Kettering Cancer Center, New York, NY 10021, USA
}

Received January 22, 2013; Accepted March 28, 2013

DOI: $10.3892 /$ or.2013.2513

\begin{abstract}
Rhabdomyosarcoma (RMS) is a soft tissue sarcoma categorized into two major subtypes: alveolar RMS (ARMS) and embryonal RMS (ERMS). Most ARMS express the PAX3-FOXO1 (P3F) fusion oncoprotein generated by the $2 ; 13$ chromosomal translocation. In the present study, the downstream target genes of P3F were identified by analyzing two independent sets of gene expression profiles: primary RMS tumors and RD ERMS cells transduced with inducible P3F constructs. We found 34 potential target genes (27 upregulated and 7 downregulated) that were significantly and differentially expressed between P3F-positive and P3F-negative categories, both in primary RMS tumors and in the inducible P3F cell culture system. Gene ontology analysis of microarray data of the inducible P3F cell culture system employed indicated apoptosis, cell death, development, and signal transduction as overrepresented significant functional categories found in both upregulated and downregulated genes. Therefore, among the 34 potential target genes, the expression of cell death-related [Gremlin1, cysteine knot superfamily 1, BMP antagonist 1 (GREM1) and death-associated protein kinase 1 (DAPK1)] and development-related [myogenic differentiation 1 (MYOD1) and hairy/enhancer-of-split related with YRPW motif 1 (HEY1)] genes were further investigated. The differential expression of GREM1, DAPK1, MYOD1 and HEY1 was confirmed in
\end{abstract}

Correspondence to: Dr Eun Hyun Ahn, Department of Pathology, University of Washington School of Medicine, Box 357705, 1959 NE Pacific St., Seattle, WA 98195, USA

E-mail: ahneun@uw.edu; ahneun@gmail.com

Present addresses: ${ }^{5}$ National Institute of Genomics, Mexico City, 01900 Mexico; ${ }^{6}$ Department of Tumor Biology, Curie Institute, Paris, France

Abbreviations: RMS, rhabdomyosarcoma; Q, glutamine; ARMS, alveolar rhabdomyosarcoma; ERMS, embryonal rhabdomyosarcoma; ER, estrogen receptor; pK, pK1; P3F, PAX3-FOXO1; P3F-ER, PAX3-FOXO1-ER; Tmf, 4-hydroxytamoxifen

Key words: rabdomyosarcoma, PAX3-FOXO1, gene expression, microarray independent tumors and inducible cell culture systems. The expression of GREM1, DAPK1 and MYOD1 were significantly upregulated; HEY1 was significantly downregulated in independent P3F-positive ARMS tumors and transcriptionally active P3F cells, compared to those in ERMS tumors and transcriptionally inactive P3F cells. This study identified target genes of P3F and suggested that four downstream targets (GREM1, DAPK1, MYOD1 and HEY1) can contribute to the biological activities of $\mathrm{P} 3 \mathrm{~F}$ involved in growth suppression or cell death and myogenic differentiation.

\section{Introduction}

Rhabdomyosarcoma (RMS), the most common type of pediatric soft tissue sarcoma, is associated with the skeletal muscle lineage. Pediatric RMS is categorized into two major subtypes: embryonal RMS (ERMS) and alveolar RMS (ARMS) based on their histologic appearance (1). ERMS shows a more favorable prognosis, while ARMS is more aggressive with a high frequency of metastases at initial diagnosis and a worse prognosis $(2,3)$.

ERMS has not been found to be associated with any diagnostic genetic alterations, but loss of heterozygosity at $11 \mathrm{p} 15$ is a common finding $(4,5)$. In contrast, ARMS is associated with recurrent chromosomal translocations. The translocation $\mathrm{t}(2 ; 13)(\mathrm{q} 35 ; \mathrm{q} 14)$ leading to the PAX3-FOXO1 (P3F) gene fusion was found to be present in 55\% of ARMS cases, while the translocation $\mathrm{t}(1 ; 13)(\mathrm{q} 36 ; \mathrm{q} 14)$ leading to the PAX7-FOXO1 gene fusion was present in $22 \%$ of cases, and $23 \%$ of ARMS were fusion-negative (2). ARMS with the 2;13 translocation is characterized as having overexpression of $\mathrm{P} 3 \mathrm{~F}$ relative to wild-type PAX3, at both the RNA and protein levels (6). In both PAX3 and P3F, an alternative splice occurs at the intron 2 /exon 3 junction resulting in inclusion or exclusion of a glutamine $(\mathrm{Q})$ residue $[\mathrm{PAX} 3(+\mathrm{Q})$ or $(-\mathrm{Q})$ forms, respectively] (7). As a result, PAX3(+Q)-FOXO1 and PAX3(-Q)-FOXO1 are coexpressed in P3F-positive ARMS tumors.

Among RMS tumors, PAX3(+/-Q)-FOXO1-positive ARMS is the most clinically intractable fusion subtype of pediatric RMS $(1,2,8,9)$. However, the histologic classification of RMS into ARMS and ERMS may be difficult in some cases (9) and there are no specific drugs for treating specific 
histologic or fusion subtypes. Therefore, there is substantial impetus to elucidate target genes of $\mathrm{P} 3 \mathrm{~F}$, which can be used to identify therapeutic targets and markers for use in RMS diagnosis and management.

Although several recent studies utilized gene expression profiles to classify RMS and/or identify target genes of P3F and PAX7-FOXO1 or P3F only in ARMS (10-16), more research is needed to validate the function of genes that are biologically relevant in ARMS development.

In the present study, we identified potential target genes of P3F by analyzing gene expression profiles from two independent systems: primary tumors (P3F-positive ARMS and fusion-negative ERMS) and a cell culture system expressing the inducible PAX3(+/-Q)-FOXO1-estrogen receptor (ER) ligand binding domain construct in the pBabe $(\mathrm{pB})$ retroviral vector. Among the potential target genes of $\mathrm{P} 3 \mathrm{~F}$, we focused on cell death or apoptosis-related [Gremlin1, cysteine knot superfamily 1, BMP antagonist 1 (GREM1), death-associated protein kinase 1 (DAPK1)] and development-related [myogenic differentiation 1 (MYOD1) and hairy/enhancer-of-split related with YRPW motif 1 (HEY1)] genes since apoptosis and development were significantly overrepresented functional categories in our gene expression profiles.

\section{Materials and methods}

Tumor samples. The tumor specimens (15 ERMS and 16 P3F-positive ARMS) used for microarray were previously described (15). Independent tumor specimens (20 ERMS and 17 P3F-positive ARMS) examined for quantitative reversetranscription PCR (qRT-PCR) in the validation studies were previously described (15). The presence of P3F in ARMS tumor specimens was determined and confirmed by RT-PCR and/or qRT-PCR (17).

Cell culture. RD ERMS cell line and RD-derived cells transduced with inducible PAX3(+/-Q)-FOXO1 in $\mathrm{pB}$ or pK1 (pK) were maintained in Dulbecco's modified Eagle's medium (DMEM) (Invitrogen, Grand Island, NY, USA) with $10 \%$ fetal bovine serum (FBS) (Thermo Scientific HyClone, Logan, UT, USA). The medium was supplemented with $1 \%$ penicillin/streptomycin $(\mathrm{P} / \mathrm{S})$ and $1 \%$ antibiotic-antimycotic (AM) (both from Invitrogen).

DNA constructs and construction of PAX3(-Q)-FOXO1-ER in $p B$ retroviral vector. Retroviral constructs with several relevant DNA inserts [pK1-PAX3(+Q)-FOXO1-ER (pK-P3F-ER), pBabe-PAX2(+Q)-FOXO1-ER (pB-P3F-ER) and pCDNA3PAX3(+Q)-FOXO1-ER (pCDNA-P3F-ER)] were previously generated by Dr Frederic G. Barr's laboratory (18-21). A modified ER ligand-binding domain was provided by $\mathrm{Dr}$ G.I. Evan (22). Unless it is noted as PAX3(-Q)-FOXO1, the P3F constructs used in our studies refer to the PAX3(+Q)-FOXO1 isoform. In order to clone an inducible construct of PAX3(-Q)-FOXO1-ER into the $\mathrm{pB}$ retroviral vector, two consecutive mutations were introduced, using QuikChange site-directed mutagenesis (Stratagene; Agilent Technologies, Santa Clara, CA, USA) followed by multiple subcloning steps. Protocols are available upon request.
Establishment of ERMS cell culture systems inducibly expressing $P 3 F$. Retroviral transduction was performed as described previously with modifications $(20,21)$. Cells that were transduced with retroviral DNA constructs were selected with puromycin (BD Biosciences, San Jose, CA, USA) at $1 \mu \mathrm{g} / \mathrm{ml}$ for RD-derived cells with PAX3(+/-Q)-FOXO1-ER in $\mathrm{pB}$ or in pK1. Cells carrying inducible PAX3(+/-Q)-FOXO1-ER were treated with the ligand 4-hydroxytamoxifen (Tmf) (SigmaAldrich, St. Louis, MO, USA), which activates and induces transcriptional activity of PAX3(+/-Q)-FOXO1-ER by translocating PAX3(+/-Q)-FOXO1-ER to the nucleus.

Luciferase reporter assays. RD cells expressing PAX3(+/-Q)-FOXO1-ER in $\mathrm{pB}$ or $\mathrm{pB}$ alone were plated at $5 \times 10^{4}$ per well in $24-w e l l$ plates. The cells were transfected with $0.3 \mu \mathrm{g}$ firefly luciferase reporter DNA containing PAX3 DNA binding sites (6 x PRS9) and $0.015 \mu \mathrm{g}$ pRL-TK Renilla luciferase DNA using FuGENE ${ }^{\circledR} 6$ (both from Promega, Madison, WI, USA) and incubated for 2 days. The transcriptional activity of PAX3(+/-Q)-FOXO1 was measured by the dual-luciferase assay (Promega) and was normalized by transfection efficiency control (pRL-TK Renilla luciferase).

RNA extraction and microarray data analysis. For microarray analysis, total RNA was extracted using the RNeasy Mini Kit (Qiagen, Valencia, CA, USA). For other studies including qRT-PCR and RT-PCR, total RNA was extracted using RNA STAT-60 (Tel-Test, Inc., Friendswood, TX, USA). Microarray analysis of RNA isolated from RMS tumors and RD cell culture systems expressing inducible PAX3(+/-Q)-FOXO1-ER in $\mathrm{pB}$ was performed on Affymetrix arrays. Microarray analysis of 31 RNA tumor samples (15 ERMS and 16 P3F-positive ARMS) was described in our previous publication (15).

For microarray analysis of inducible cell culture systems, three RD cell populations were independently transduced with each construct [PAX3(+Q)-FOXO1-ER in pB; PAX3(-Q)-FOXO1-ER in $\mathrm{pB}$; $\mathrm{pB}$ vector alone] and treated without or with $30 \mathrm{nM}$ Tmf for $24 \mathrm{~h}$ to generate a total of 18 samples. RNA isolated from these cells was hybridized onto Affymetrix GeneChip Human Genome U133 Plus v.2.0 (HG U133+ v.2.0). After the expression levels were determined using Affymetrix Microarray Suite 5.0, the expression levels were normalized in GeneSpring (Agilent Technology). The probe-sets, whose expression levels were detected in at least 2 of the 18 samples (in inducible cell culture system) or 1 of the 31 samples (in tumors), were selected for further analysis. To compare the gene expression profiles from tumors with those from the inducible cell culture systems, the probes on the HG U133A array were used for GeneSpring analysis.

The Significance Analysis of Microarrays (SAM) (23) with two-class paired condition was used as the common method to identify differentially expressed genes between $16 \mathrm{P} 3 \mathrm{~F}$ positiveARMS and 15 fusion-negative ERMS tumors as well as between the RD cells expressing PAX3(+/-Q)-FOXO1-ER in pB (treated with Tmf; 6 samples) and the RD cells expressing PAX3(+/Q)-FOXO1-ER in pB (not treated with Tmf; 6 samples). Data was filtered by a false discovery rate (FDR) at $<5 \%$ (FDR $4.94 \%$ for cells; FDR $4.91 \%$ for tumors) and $\geq 1.5$ fold-change. The PAX3(+Q)-FOXO1-ER and PAX3(-Q)-FOXO1-ER gene expression profiles were pooled since SAM did not reveal 
significant differentially expressed genes between these two groups. The genes that were differentially expressed between transcriptionally active PAX3(+/-Q)-FOXO1-ER (Tmf-treated) and inactive PAX3(+/-Q)-FOXO1-ER (untreated control) were then examined.

Finally, common genes that were present in both tumors and the inducible PAX3(+/-Q)-FOXO1-ER cell culture system were identified by Venn Diagram analysis. After this analysis, the probe-sets that encoded the same gene with similar expression profiles and showed raw expression values at $<150$ were removed. Classifications and functional annotations of genes were analyzed via web-accessible programs: Expression Analysis Systematic Explorer (EASE) 2010 and Database for Annotation, Visualization and Integrated Discovery (DAVID) 2010 (24).

Quantitative reverse transcription- $P C R(q R T-P C R)$ analysis. The qRT-PCR assay was performed as described previously $(15,16)$. Test gene assays were normalized to the expression of 18S rRNA. Taqman gene expression assays used (Applied Biosystems) were: DAPK1 (assay ID\# Hs00234489_ m1), GREM1 (assay ID\# Hs00171951_m1) and MYOD1 (assay ID\# Hs00159528_m1). The sequences of forward and reverse primers and probes of PAX3-FOXO1 and HEY1 are available upon request.

Extraction of cellular proteins and isolation of secreted proteins from the medium of cultured cells and immunoblot analysis. The cells were seeded at $10^{6} / 100-\mathrm{mm}$ dish in phenol red-free DMEM medium containing 10\% FBS, $1 \% \mathrm{P} / \mathrm{S}$ and $1 \% \mathrm{AM}$ for $24 \mathrm{~h}$ prior to Tmf treatment. The cells expressing inducible PAX3(+/-Q)-FOXO1-ER were then treated with $30 \mathrm{nM}$ Tmf for various times. Cellular proteins were lysed as described previously (20). To assay the secreted GREM1 protein, the medium containing 10\% FBS was replaced with FBS-free DMEM after $24 \mathrm{~h}$ of Tmf treatment, and cells were cultured for an additional 24 or $48 \mathrm{~h}$ with Tmf treatment. The medium of the cultured cells was collected and concentrated to $<1,000 \mu 1$ using Amicon Ultra-4 with Ultracel-10K (UFC80-1024; Millipore, Billerica, MA, USA). Protein was quantified using Coomassie Plus Protein Assay (Pierce Biotechnology, Inc., Rockford, IL, USA).

Immunoblot analysis was performed as described previously (25) with modifications. The primary antibodies used were: anti-PAX3 rabbit polyclonal, DAPK1 (ab10443; both from Abcam, Cambridge, MA, USA) and GREM1 (cat\# AP6133a; Abgent, San Diego, CA, USA).

Statistical analysis. The qRT-PCR data of tumors (20 fusionnegative ERMS and 17 P3(+Q)F-positive ARMS) were analyzed by the Mann-Whitney Test (Wilcoxon Rank Sum Test) and differences between the two tumor groups were considered significant at a P-value $<0.05$. Statistical analysis of gene expression profiles are described in 'RNA extraction and microarray data analysis'.

\section{Results}

Development of inducible PAX3(+/-Q)-FOXO1 cell culture systems. RD ERMS cell line was transduced with

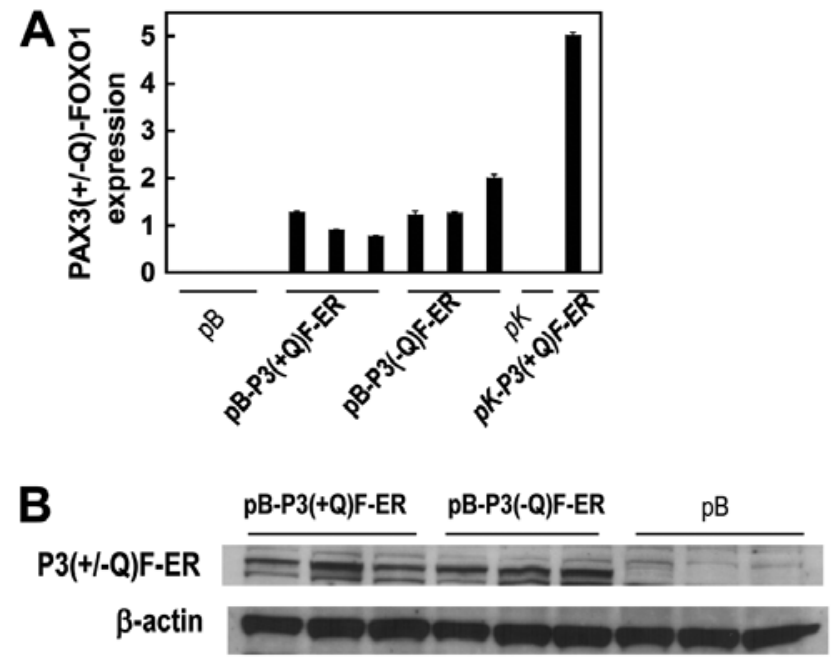

Figure 1. Expression of inducible forms of PAX3(+/-Q)-FOXO1 in RD cells transduced with pBabe (pB), pB-PAX3(+Q)FKHR-ER [pB-P3(+Q)F-ER], pB-PAX3(-Q)FKHR-ER [pB-P3(-Q)F-ER], pK1 (pK), or pK-PAX3(+Q)FOXO1-ER [pK-P3(+Q)F-ER]. (A) Total RNA was isolated and the expression of PAX3(+/-Q)-FOXO1 was determined by qRT-PCR and was normalized to $18 \mathrm{~S}$ rRNA expression (means $\pm \mathrm{SD}$ ). (B) Total protein was extracted after 2 weeks of puromycin selection from RD ERMS cells transduced with pB-P3(+Q)F-ER (lanes 1-3), pB-P3(-Q)F-ER (lanes 4-6), $\mathrm{pB}$ vector alone (lanes 7-9).

PAX3(+/-Q)-FOXO1-ER in pB [pB-P3(+/-Q)F-ER] or pB alone and selected with puromycin for 2 weeks. The transduction of $\mathrm{RD}$ cells with these $\mathrm{pB}-\mathrm{P} 3(+/-\mathrm{Q}) \mathrm{F}-\mathrm{ER}$ constructs resulted in the expression of PAX3(+/-Q)-FOXO1-ER at both the mRNA (Fig. 1A) and protein levels (Fig. 1B).

In order to determine and confirm the inducible PAX3(+/-Q)-FOXO1 function, the RD-derived cells transduced with $\mathrm{pB}-\mathrm{P} 3(+/-\mathrm{Q}) \mathrm{F}-\mathrm{ER}$ or $\mathrm{pB}$ were transiently transfected with a luciferase gene reporter construct containing PAX3 DNA binding sites and were treated with tamoxifen at various concentrations $(0,1,3,10,30$ and $100 \mathrm{nM})$ for $24 \mathrm{~h}$ (Fig. 2). The RD cells transduced with an empty vector $\mathrm{pB}$ alone (Fig. 2A) showed a very low ( 0.02) transcriptional activity indicating only an endogenous PAX3 transcriptional activity. In contrast, $R D$ cells transduced with $\mathrm{pB}-\mathrm{P} 3(+\mathrm{Q}) \mathrm{F}-\mathrm{ER}$ (Fig. 2B) or pB-P3(-Q)F-ER (Fig. 2C) demonstrated much higher transcriptional activities as Tmf concentrations increased. The maximal transcriptional activity in RD-derived cells with pB-P3(+/-Q)F-ER was observed at $30 \mathrm{nM}$ Tmf and showed a $\sim 46$ - to 47 -fold difference in luciferase gene transactivation between RD-P3(+/-Q)F-ER cells and RD-pB cells (Fig. 2B and C).

Microarray data analysis of tumors and inducible cell culture system identifies 34 potential target genes of P3F. To identify target genes of $\mathrm{P} 3 \mathrm{~F}$ in the present study, we utilized RD cells expressing inducible PAX3(+/-Q)-FOXO1-ER constructs in the $\mathrm{pB}$ vector (Figs. 1 and 2) based on the following rationale and significance. First, because the expression of PAX3(+/-Q)-FOXO1 in ARMS tumors varies, the utilization of cell culture models having various levels of PAX3(+/-Q)-FOXO1 can provide an unbiased approach for identifying potential target genes of PAX3(+/-Q)-FOXO1. Our previous gene expression profiling study investigated RD 

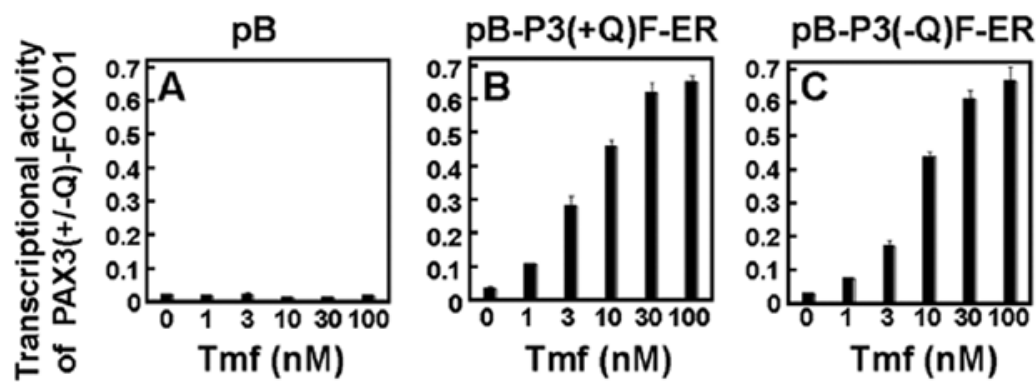

Figure 2. Transcriptional activity of inducible forms of PAX3-FOXO1 (P3F) in RD cells transduced with (A) pBabe (pB), (B) pB-PAX3(+Q)FKHR-ER [pB-P3(+Q)F-ER], or (C) pB-PAX3(-Q)FKHR-ER [pB-P3(-Q)F-ER]. The cells were seeded at $5 \times 10^{4}$ cells/well, and transcriptional activity was determined by dual luciferase assay with a reporter containing PAX3 DNA binding sites (6 x PRS9) and then the cells were treated with tamoxifen at 1,3,10,30 and 100 nM for $24 \mathrm{~h}$. The luciferase activity was normalized by transfection efficiency control (pRL-TK-Renilla). Data shown are means \pm SEM ( $\mathrm{n}=3$ ). Where an error bar is not shown, it lies within the dimensions of the symbol.

cells transduced with an inducible PAX3(+Q)-FOXO1-ER construct in the $\mathrm{pK}$ vector that enforces high expression of PAX3(+Q)-FOXO1-ER (16). In contrast, our present expression profiling study used the $\mathrm{pB}$ vector to express lower levels of inducible PAX3(+/-Q)-FOXO1-ER in RD cells. Second, a previous expression profiling analysis of the inducible cell system (16) was complicated by the confounding factor of a treatment-day effect. Thus, microarray data from inducible cells with PAX3(+Q)-FOXO1-ER in pK were analyzed by Mixed ANOVA, whereas primary RMS tumors were analyzed by SAM. In comparison, no such confounding factors were present in the present study and thus microarray data from both primary RMS tumors and inducible cells were both analyzed by SAM.

After analyzing microarray data from primary RMS tumors as well as inducible cells [PAX3(+/-Q)-FOXO1-ER in pB] by SAM, data were filtered for expression values, FDR, and fold-change (FDR at $<5 \%$ and $\geq 1.5$ fold-change). In the tumor microarray data, 1,693 probe-sets (1,115 upregulated and 578 downregulated) on the HG U133A platform were either significantly upregulated or downregulated in the PAX3(+/-Q)-FOXO1-positive ARMS tumors $(\mathrm{n}=16)$ compared to the fusion-negative ERMS tumors $(\mathrm{n}=15)$. Analysis of genes differentially expressed between induced (transcriptionally active) PAX3(+/-Q)-FOXO1-ER in $\mathrm{pB}(\mathrm{Tmf}$ treated, $\mathrm{n}=6)$ and uninduced PAX3(+/-Q)-FOXO1-ER in pB (untreated control, $\mathrm{n}=6$ ) revealed 540 probe sets (304 upregulated and 236 downregulated) on the HG U133+ v.2.0 array. Of these 540 probe sets, 328 were present on the HGU133A array (182 upregulated and 146 downregulated). Finally, 34 potential target genes (27 upregulated and 7 downregulated) were differentially expressed both in tumors and in the inducible cell culture system, using probes from the HG U133A array (Table I).

Many of the differentially expressed genes in the PAX3(+/-Q)-FOXO1-ER cell systems are related to apoptosis and development. To investigate biological consequences of the PAX3(+/-Q)-FOXO1 expression signature, the 540 significantly upregulated or downregulated probes identified in $\mathrm{RD}$ cells expressing the inducible PAX3(+/-Q)-FOXO1-ER in $\mathrm{pB}$ on the HG-U133+ v.2.0 platform were analyzed using EASE and DAVID (24). Notably, 10 out of the 15 most significant functional categories analyzed by EASE for the upregulated genes were apoptosis, cell death, or negative regulation of cell proliferation (Fig. 3).

The most significant 20 overrepresented functional groups in the RD cells carrying inducible PAX3(+/-Q)-FOXO1-ER in pB identified by DAVID are listed in Table II. Among these 20 groups, five groups were commonly found in both upregulated and downregulated genes. Four out of these 5 functional groups were related to development (developmental process, multicellular organismal development, anatomical structure development, system development) and one group to signal transduction. MYODI (an upregulated gene) and HEYl (a downregulated gene) were found in all of the 5 common functional groups.

GREM1, DAPK1, MYOD1 and HEY1 are significantly and differentially expressed in both independent tumors and inducible PAX3-FOXO1-ER cell systems. Since gene ontology analysis EASE or DAVID indicated apoptosis (Fig. 3) and development (Table II) as significant functional categories, we validated the expression of apoptosis-related genes (GREMI, $D A P K 1)$ and development-related genes (MYODI, HEY1) that were identified among the 34 potential target genes (Table I) differentially expressed in both primary tumors and the inducible cell culture system. These genes were analyzed in an independent panel of primary tumors (20 fusion-negative ERMS and 17 PAX3-FOXO1-positive ARMS) (Fig. 4; Table III) and RD cells carrying inducible PAX3(+/-Q)FOXO1-ER in $\mathrm{pB}$ or $\mathrm{pK}$ (Tmf-treated versus untreated control) (Fig. 5; Table III). The validation was carried out at the RNA level using qRT-PCR for the expression of GREMI (Figs. 4A and 5A), DAPK1 (Figs. 4B and 5B), MYODI (Figs. 4C and 5C) and HEYl (Figs. 4D and 5D). Furthermore, the upregulation of GREM1 and DAPK1 at the protein level was validated using immunoblot analysis (Fig. 5E). These validation studies of GREM1, DAPK1, MYOD1 and HEY1 (Figs. 4 and 5) consistently demonstrated significant differential expression, which was comparable to that noted in the microarray expression signature (Table III).

\section{Discussion}

In the present study, we developed the RD ERMS cell culture system expressing the inducible PAX3(+/-Q)-FOXO1-ER 
Table I. Potential target genes of PAX3-FOXO1 as identified by microarray analysis.

\begin{tabular}{|c|c|c|c|c|}
\hline \multirow[b]{2}{*}{ Affymetrix ID } & \multirow[b]{2}{*}{ Symbol } & \multirow[b]{2}{*}{ Gene name } & \multicolumn{2}{|c|}{ Fold-change } \\
\hline & & & $\begin{array}{l}\text { Tumors } \\
(\mathrm{n}=31)\end{array}$ & $\begin{array}{l}\text { Inducible cells } \\
\qquad(\mathrm{n}=12)\end{array}$ \\
\hline \multicolumn{5}{|c|}{ Downregulated genes in P3F-positive categories of tumors and cells } \\
\hline 218974_at & FLJ10159 & Hypothetical protein FLJ10159 & 2.85 & 1.82 \\
\hline 218839_at & HEY1 & Hairy/enhancer-of-split related with YRPW motif 1 & 6.21 & 1.72 \\
\hline 209030_s_at & IGSF4 & $\begin{array}{l}\text { Cell adhesion molecule } 1 \\
\text { (Immunoglobulin superfamily, member } 4 \text { ) }\end{array}$ & 2.86 & 1.53 \\
\hline 203708_at & PDE4B & $\begin{array}{l}\text { Phosphodiesterase 4B, cAMP-specific } \\
\text { (phosphodiesterase E4 dunce homolog, Drosophila) }\end{array}$ & 4.80 & 3.77 \\
\hline 202732_at & PKIG & Protein kinase (cAMP-dependent, catalytic) inhibitor $\gamma$ & 1.85 & 1.69 \\
\hline 209875_s_at & SPP1 & $\begin{array}{l}\text { Secreted phosphoprotein } 1 \text { (osteopontin, } \\
\text { bone sialoprotein I, early T-lymphocyte activation 1) }\end{array}$ & 6.43 & 2.14 \\
\hline 201368_at & ZFP36L2 & Human Tis11d gene, complete cds. & 2.60 & 1.56 \\
\hline \multicolumn{5}{|c|}{ Upregulated genes in P3F-positive categories of tumors and cells } \\
\hline 209459_s_at & ABAT & 4-Aminobutyrate aminotransferase & 10.52 & 5.84 \\
\hline 206704_at & CLCN5 & $\begin{array}{l}\text { Chloride channel } 5 \text { (nephrolithiasis } 2 \text {, } \\
\text { X-linked, Dent disease) }\end{array}$ & 2.68 & 2.01 \\
\hline 203139_at & DAPK1 & Death-associated protein kinase 1 & 2.51 & 2.40 \\
\hline 213712_at & ELOVL2 & Catenin (cadherin-associated protein), $\alpha$-like 1 & 4.16 & 1.92 \\
\hline 218469_at & GREM1 & Gremlin1, cysteine knot superfamily 1, BMP antagonist 1 & 5.34 & 2.13 \\
\hline 203233_at & IL4R & Interleukin 4 receptor & 2.03 & 1.74 \\
\hline 203126_at & IMPA2 & Inositol(myo)-1(or 4)-monophosphatase 2 & 2.90 & 1.92 \\
\hline 202794_at & INPP1 & Inositol polyphosphate-1-phosphatase & 1.89 & 1.51 \\
\hline 205902_at & KCNN3 & $\begin{array}{l}\text { Potassium intermediate/small conductance } \\
\text { calcium-activated channel, subfamily } \mathrm{N} \text {, member } 3\end{array}$ & 6.40 & 1.97 \\
\hline 204094_s_at & KIAA0669 & TSC22D2, TSC22 domain family, member 2 & 4.63 & 2.18 \\
\hline 218829_s_at & KIAA1416 & CHD7, chromodomain helicase DNA binding protein 7 & 2.26 & 2.31 \\
\hline 211042_x_at & MCAM & Melanoma cell adhesion molecule & 2.14 & 1.73 \\
\hline 213256_at & MGC48332 & Hypothetical protein MGC48332 & 4.35 & 1.52 \\
\hline 206657_s_at & MYOD1 & Myogenic differentiation 1 & 2.59 & 2.28 \\
\hline 209106_at & NCOA1 & Nuclear receptor coactivator 1 & 3.15 & 1.66 \\
\hline 209289_at & NFIB & Nuclear factor I/B & 1.97 & 1.51 \\
\hline 205858_at & NGFR & $\begin{array}{l}\text { Nerve growth factor receptor } \\
\text { (TNFR superfamily, member 16) }\end{array}$ & 4.03 & 1.77 \\
\hline 204105_s_at & NRCAM & Neuronal cell adhesion molecule & 6.62 & 2.36 \\
\hline 209123_at & QDPR & Quinoid dihydropteridine reductase & 5.04 & 1.84 \\
\hline 203217_s_at & SIAT9 & $\begin{array}{l}\text { Sialyltransferase } 9 \text { (CMP-NeuAc:lactosylceramide } \\
\alpha-2,3 \text {-sialyltransferase; GM3 synthase) }\end{array}$ & 2.43 & 1.84 \\
\hline 203625_x_at & SKP2 & S-phase kinase-associated protein $2(\mathrm{p} 45)$ & 2.64 & 1.91 \\
\hline 213624_at & SMPDL3A & Sphingomyelin phosphodiesterase, acid-like 3A & 2.05 & 1.76 \\
\hline 212761_at & TCF7L2 & Transcription factor 7-like 2 (T-cell specific, HMG-box) & 2.68 & 1.59 \\
\hline 209656_s_at & TM4SF10 & Transmembrane 4 superfamily member 10 & 2.36 & 3.11 \\
\hline 215389_s_at & TNNT2 & Troponin $\mathrm{T} 2$, cardiac & 5.39 & 1.86 \\
\hline 219038_at & ZCWCC2 & Zinc finger, CW-type with coiled-coil domain 2 & 3.52 & 2.02 \\
\hline 49111_at & DKFZp762M127 & $\begin{array}{l}\text { MRNA; cDNA DKFZp762M127 } \\
\text { (from clone DKFZp762M127) }\end{array}$ & 6.60 & 8.23 \\
\hline
\end{tabular}

The 34 genes were significantly differentially expressed between P3F positive-ARMS and fusion-negative ERMS tumors and between induced (transcriptionally active) PAX3(+/-Q)-FOXO1-ER in $\mathrm{pB}$ and uninduced (inactive) PAX3(+/-Q)-FOXO1-ER in pB in RD-derived cells. The fold-change difference was calculated by dividing the expression levels of P3F-positive categories of tumors (ARMS) and inducible cells with those of P3F-negative categories of tumors (ERMS) and inducible cells. P3F, PAX3-FOXO1; ARMS, alveolar rhabdomyosarcoma; ERMS, embryonal rhabdomyosarcoma; $\mathrm{pB}, \mathrm{pBabe}$. 
Table II. DAVID analysis of the microarray data of the inducible PAX3-FOXO1 cell culture system.

Gene category
$\begin{gathered}\text { Counts } \\ \text { (no. of genes) }\end{gathered}$

Upregulated genes in transcriptionally active PAX3(+/-Q)-FOXO1 cells

Protein binding

Developmental process

$\begin{array}{rrr}112 & 163 & 1.08 \mathrm{E}-08 \\ 62 & 91 & 3.04 \mathrm{E}-06 \\ 44 & 63 & 2.60 \mathrm{E}-04 \\ 6 & 7 & 4.32 \mathrm{E}-04 \\ 11 & 19 & 5.41 \mathrm{E}-04 \\ 40 & 65 & 6.75 \mathrm{E}-04 \\ 6 & 7 & 7.56 \mathrm{E}-04 \\ 6 & 7 & 1.24 \mathrm{E}-03 \\ 67 & 96 & 1.48 \mathrm{E}-03 \\ 151 & 209 & 1.49 \mathrm{E}-03 \\ 63 & 94 & 1.88 \mathrm{E}-03 \\ 10 & 14 & 2.09 \mathrm{E}-03 \\ 33 & 50 & 2.13 \mathrm{E}-03 \\ 62 & 96 & 3.27 \mathrm{E}-03 \\ 4 & 5 & 3.68 \mathrm{E}-03 \\ 69 & 99 & 3.94 \mathrm{E}-03 \\ 12 & 19 & 4.11 \mathrm{E}-03 \\ 57 & 84 & 4.36 \mathrm{E}-03 \\ 10 & 12 & 4.43 \mathrm{E}-03 \\ 10 & 14 & 4.88 \mathrm{E}-03\end{array}$

Multicellular organismal development

Ras GTPase binding

Enzyme binding

Anatomical structure development

Small GTPase binding

GTPase binding

Regulation of cellular process

Binding

Cell communication

Central nervous system development

System development

Splice variant

Glycolipid metabolic process

Regulation of biological process

Transcription factor binding

Signal transduction

Apoptosis

Transcription cofactor activity

Downregulated genes in transcriptionally active PAX3(+/-Q)-FOXO1 cells

Immune system process

$\begin{array}{rrr}26 & 36 & 5.41 \mathrm{E}-05 \\ 57 & 77 & 5.48 \mathrm{E}-05 \\ 25 & 35 & 9.55 \mathrm{E}-05 \\ 50 & 67 & 1.39 \mathrm{E}-04 \\ 37 & 50 & 1.97 \mathrm{E}-04 \\ 39 & 53 & 2.46 \mathrm{E}-04 \\ 7 & 11 & 2.99 \mathrm{E}-04 \\ 5 & 8 & 3.21 \mathrm{E}-04 \\ 21 & 31 & 3.59 \mathrm{E}-04 \\ 3 & 5 & 4.45 \mathrm{E}-04 \\ 24 & 35 & 4.58 \mathrm{E}-04 \\ 44 & 60 & 5.68 \mathrm{E}-04 \\ 53 & 68 & 6.54 \mathrm{E}-04 \\ 3 & 5 & 7.37 \mathrm{E}-04 \\ 5 & 8 & 8.24 \mathrm{E}-04 \\ 16 & 23 & 8.50 \mathrm{E}-04 \\ 3 & 5 & 9.61 \mathrm{E}-04 \\ 6 & 6 & 1.05 \mathrm{E}-03 \\ 30 & 39 & 1.21 \mathrm{E}-03 \\ 52 & 70 & 1.22 \mathrm{E}-03\end{array}$

Glycoprotein

Anatomical structure morphogenesis

Developmental process

Anatomical structure development

Multicellular organismal development

Response to virus

von Willebrand factor, type $\mathrm{C}$

Immune response

2-5-Oligoadenylate synthetase

Negative regulation of biological process

Signal

Signal transduction

2-5-Oligoadenylate synthetase, ubiquitin like region

VWC (von Willebrand factor (vWF) type C domain)

Response to external stimulus

PIRSF005680:Interferon-induced 56K protein

Morphogenesis of an epithelium

System development

52

.41E-05

$55 \mathrm{E}-05$

Multicellular organismal process

The 540 probe sets of significantly upregulated or downregulated probes identified in RD cells expressing pB-PAX3(+/-Q)-FOXO1-ER in the HG-U133+ v.2.0 platform were analyzed using DAVID. The most significant 20 functional groups for either upregulated or downregulate genes are listed. DAVID, Database for Annotation, Visualization and Integrated Discovery; pB, pBabe.

in $\mathrm{pB}$, which is useful for determining the early short-term effects of PAX3(+/-Q)-FOXO1 and for regulating the strength and duration of transcriptional activity of PAX3-FOXO1. We analyzed two independent sets of gene expression profiles: primary RMS tumors and RD ERMS cells transduced with inducible PAX3(+/-Q)-FOXO1 constructs. We found 34 poten- 


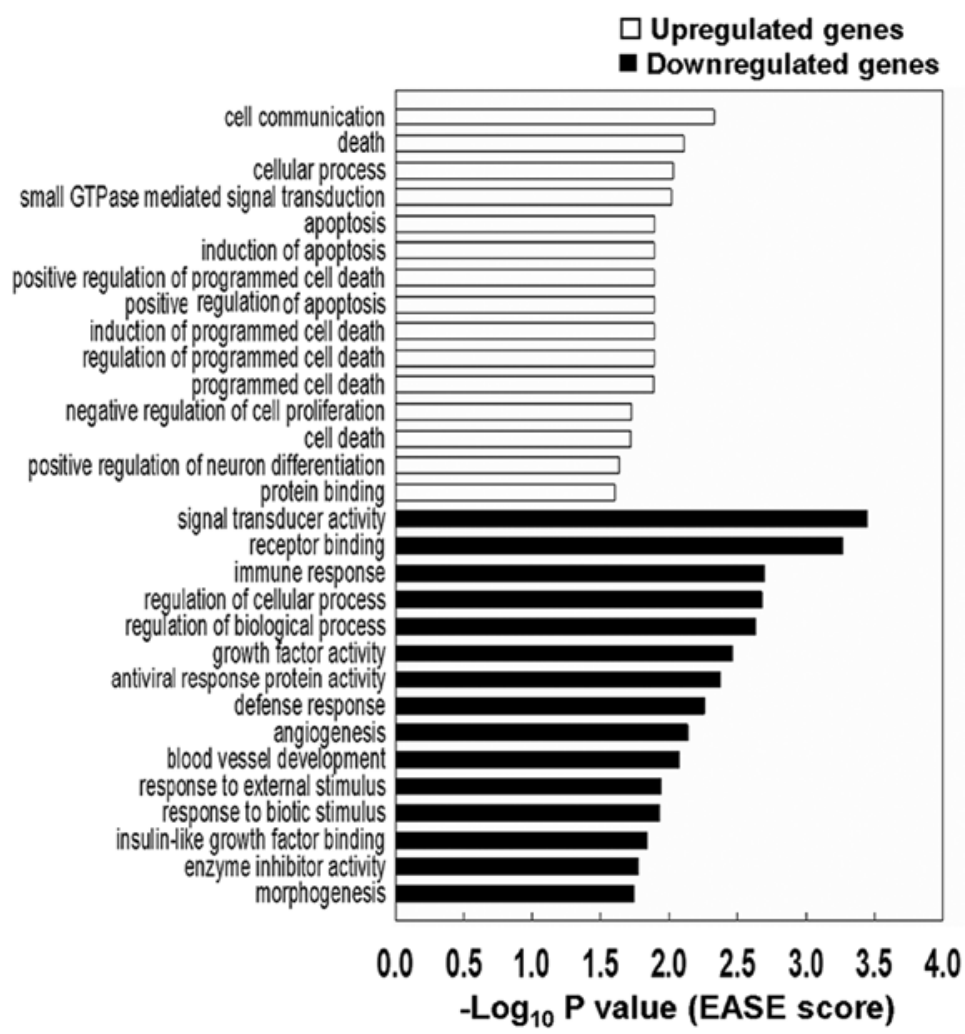

Figure 3. Expression analysis systematic explorer (EASE) of gene ontology annotations in gene expression profiles of the inducible PAX3-FOXO1 (P3F) cell systems. Functional categories enriched in the significantly upregulated ( $\square$ ) and downregulated ( $\square$ ) genes in the gene expression profiles of inducible P3F cell systems are shown.
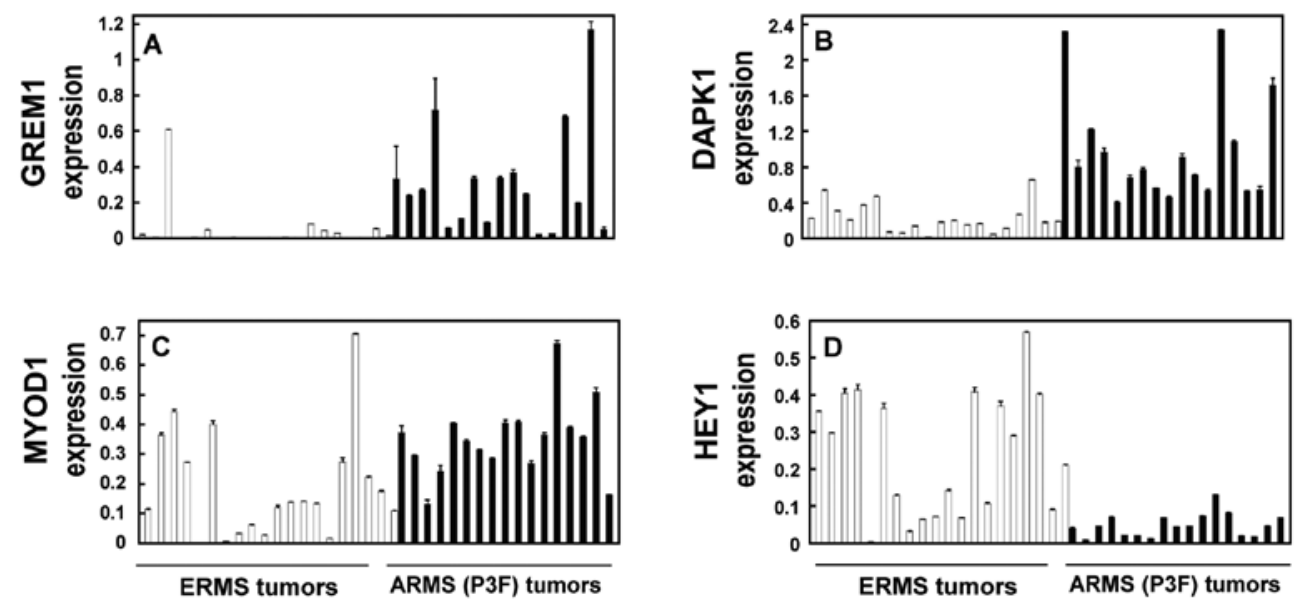

Figure 4. Validation of expression of genes in an independent set of tumors using qRT-PCR. The expression of GREM1, DAPK1, MYOD1 and HEY1 was determined by qRT-PCR in fusion-negative ERMS $(\mathrm{n}=20, \square)$ and PAX3-FOXO1 (P3F)-positive ARMS $(\mathrm{n}=17, \mathbf{\square})$ primary tumors. Total RNA was isolated and the expression levels of these genes were normalized to $18 \mathrm{~S}$ rRNA expression (mean $\pm \mathrm{SD}$ ). The mean expression for the 4 genes in P3F-positive ARMS were significantly different $(\mathrm{P}<0.005$; see Table I) from those of the ERMS, respectively.

tial target genes (27 upregulated and 7 downregulated) that were significantly and differentially expressed between PAX3(+/-Q)-FOXO1-positive and fusion-negative categories, in both primary tumors and the inducible PAX3(+/-Q)-FOXO1 cell culture system. Among the 34 genes, we investigated cell death or apoptosis-related (GREMI,DAPKI) and development-related genes (MYODI, HEYI).

Target genes of PAX3-FOXO1 and PAX7-FOXO1, or PAX3-FOXO1 only, were previously reported, based on gene expression profiling in both primary tumors and cells transduced with constitutive PAX3/PAX7-FOXO1 (13) or inducible PAX3-FOXO1-ER (16) constructs. Constitutive PAX3-FOXO1 cell system represents relatively longer (later) effects of PAX3FOXO1 compared to the inducible PAX3-FOXO1-ER cell systems. These two studies $(13,16)$ reported 81 and 39 target genes, respectively. Compared with these two previous studies, 11 and 12 target genes, respectively, were found in common with the present study (Table IV). Six genes (KCNN3, MCAM, 
Table III. Validation of the expression of 4 target genes (GREM1, DAPK1, MYOD1 and HEY1).

Fold-change

\begin{tabular}{|c|c|c|c|}
\hline & croarray & & qRT-PCR \\
\hline
\end{tabular}

\section{Downregulated genes}

HEY1

1.72

4.83

0.0000

Upregulated genes

\begin{tabular}{ll} 
GREM1 & 5.34 \\
DAPK1 & 2.51 \\
MYOD1 & 2.59 \\
\hline
\end{tabular}

2.13

6.82

0.0000

1.76

$2.51 \quad 2.40$

4.26

0.0000

2.17

MYOD1

2.28

1.86

0.0033

2.11

The expression of genes was determined by qRT-PCR in independent tumors (20 ERMS; 17 P3F-positive ARMS) and RD ERMS cells transduced with inducible PAX3(+/-Q)-FOXO1-ER in pB. For microarray, 31 tumors (15 ERMS; 16 P3F-positive ARMS) and 12 inducible cell culture samples (6 Tmf-treated, 6 untreated) were analyzed. The fold-change difference was calculated by dividing the expression levels of P3F-positive categories of tumors (ARMS) and inducible cells with those of P3F-negative categories of tumors (ERMS) and inducible cells. GREM1, gremlin1, cysteine knot superfamily 1, BMP antagonist 1; DAPK1, death-associated protein kinase 1; MYOD1, myogenic differentiation 1; HEY1, hairy/enhancer-of-split related with YRPW motif 1; qRT-PCR, quantitative reverse-transcription PCR; ERMS, embryonal rhabdomyosarcoma; P3F, PAX3-FOXO1; ARMS, alveolar rhabdomyosarcoma; pB, pBabe; Tmf, 4-hydroxytamoxifen.
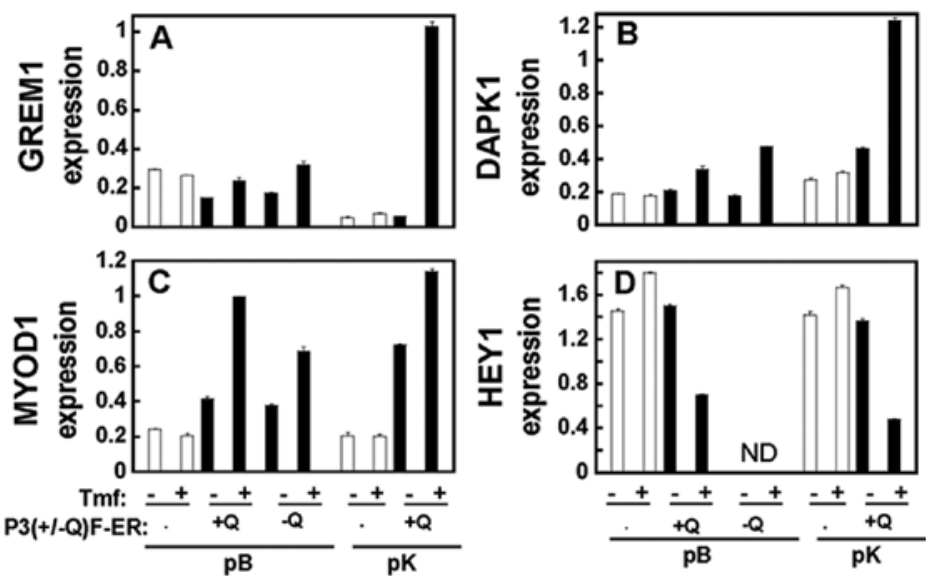

$\mathbf{E}$

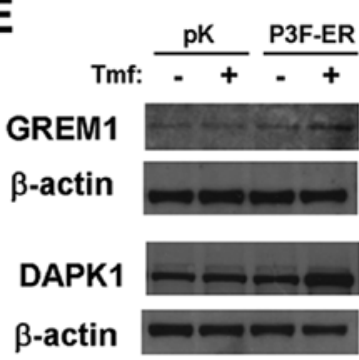

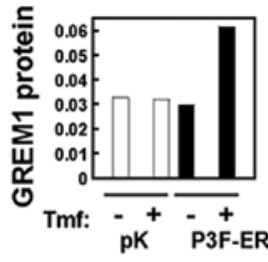

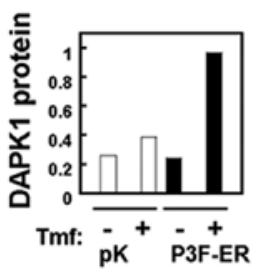

Figure 5. Validation of the expression of genes in the RD cells transduced with PAX3(+/-Q)-FOXO1-ER using qRT-PCR and immunoblot assays. (A-D) RD cells transduced with $+\mathrm{Q}$ or $-\mathrm{Q}$ isoforms of PAX3(+/Q)-FOXO1-ER [P3(+/-Q)F-ER] ( $\mathbf{\square})$ in pBabe (pB) or P3(+Q)F-ER in pK1 (pK) (घ) or retroviral vectors ( $\square$ ) alone ( $\mathrm{pB}$ or $\mathrm{pK}$ ), were treated without or with 4-hydroxytamoxifen (Tmf) at $30 \mathrm{nM}$ for $24 \mathrm{~h}$. Total RNA was isolated and the expression of these genes was determined by qRT-PCR. The relative expression levels of the 4 genes were normalized to $18 \mathrm{~S}$ rRNA (mean \pm SD; $\mathrm{n}=3$ ). (E) Cells were cultured for $48 \mathrm{~h}$ with $30 \mathrm{nM}$ Tmf. Total proteins were extracted and protein expression was determined by immunoblot analysis. The protein expression levels of GREM1 and DAPK1 were normalized by $\beta$-actin protein expression for densitometry graphs. ND, not determined.

MYOD1, TCF7L2, TM4SF10, ZFP36L2) were identified as targets in all three studies (Table IV) (13,16, present study).

We demonstrated that higher expression of MYODI and lower expression of $H E Y 1$ were consistently observed in all PAX3-FOXO1-expressing ARMS primary tumors and cells, in comparison to fusion-negative ERMS primary tumors and cells. These findings of MYODI upregulation are in accord with results reported in previous studies of MYOD1 expression in human ARMS (26,27), PAX3/PAX7-FOXO1positive cells (13), mesenchymal stem cells transfected with PAX3-FOXO1 (28), and NIH3T3 fibroblasts transduced with PAX3-FOXO1 $(10,29)$. In a previous study $(30)$, HEY1 overexpression was found to inhibit MYOD1, an early myogenic differentiation marker, which indicates that HEY1 downregulation found in our study is consistent with this PAX3-FOXO1-induced myogenic developmental program including MYOD1 upregulation. It is hypothesized that PAX3FOXO1 simultaneously reinforces myogenic determination by upregulating MYOD1, while suppressing terminal myogenic differentiation $(31,32)$.

The oncogenic activity of PAX3-FOXO1 is characterized in part by its stimulatory effects on cell proliferation (33-35) and cell survival/anti-apoptosis $(36,37)$ as well as its inhibitory role on terminal myogenic differentiation $(31,32)$. However, in addition to these effects, PAX3-FOXO1 can also cause growth suppression and cell death in other settings $(20,21,38)$. 
Table IV. Comparison of the potential target genes of PAX3-FOXO1 in three studies $(13,16$, present study).

6 common genes in all three studies

[Davicioni et al (13),

Mercado et al (16)

and present study]

12 common genes
in present study
and Mercado et al (16)

12 common genes

and Mercado et al (16)
11 common genes

in present study

and Davicioni et al (13)
16 common genes in Davicioni et al (13) and Mercado et al (16)

\begin{tabular}{llll}
\hline KCNN3 & DAPK1 & ABAT & DCX \\
MCAM & DKFZp762M127 & IL4R & DUSP4 $(\downarrow)$ \\
MYOD1 & GREM1 & INP1 & GADD45A \\
TCF7L2 & HEY1 $(\downarrow)$ & KCNN3 & IGFBP3 $(\downarrow)$ \\
TM4SF10 & KCNN3 & MCAM & KCNN3 \\
ZFP36L2 $(\downarrow)$ & MCAM & MYOD1 & MARCH3 \\
& MYOD1 & NRCAM & MCAM \\
& NCOA1 & SKP2 & MEG3 \\
& QDPR & TCF7L2 & MET \\
& TCF7L2 & TM4SF10 & MYCN \\
& TM4SF10 & ZFP36L2 $(\downarrow)$ & MYOD1 \\
& ZFP36L2 $(\downarrow)$ & & NEBL \\
& & & PRKAR2B \\
& & & TCF7L2 \\
& & & TM4SF10 \\
& & & ZFP36L2 $(\downarrow)$
\end{tabular}

The potential target genes of P3F were identified by microarray analysis in both primary RMS tumors and cells transduced with constitutive PAX3/PAX7-FOXO1 (13) or inducible P3F-ER (16, present study) constructs. Unless noted as ( $\downarrow$ ) for downregulated genes, all other genes were upregulated genes.

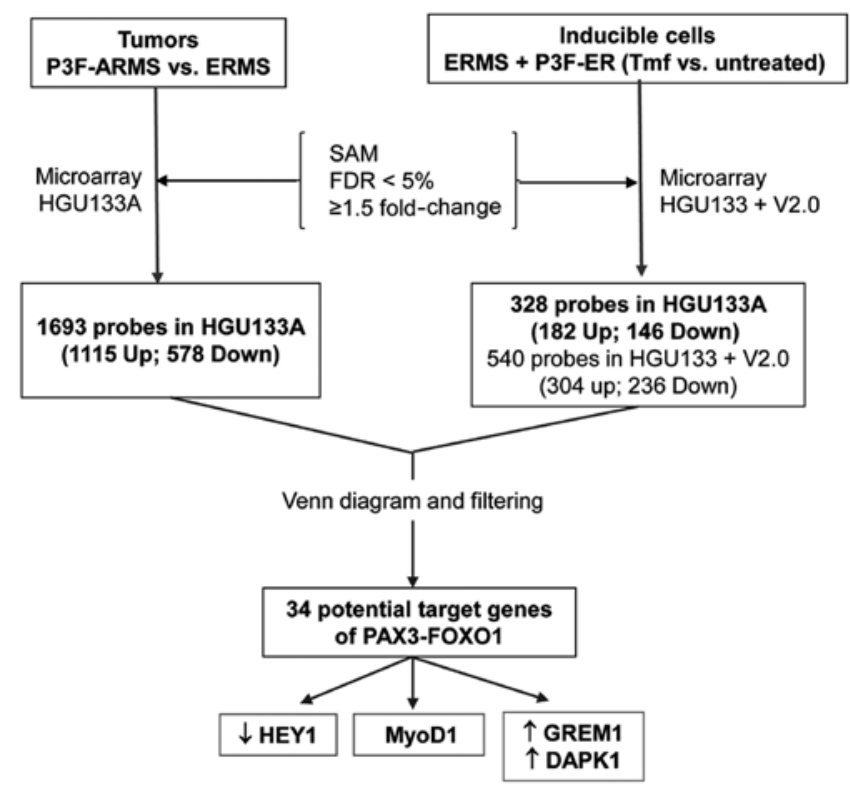

Figure 6. Identification of GREM1, DAPK1, and MYOD1 and HEY1 as potential target genes of PAX3-FOXO1 (P3F) in alveolar rhabdomyosarcoma (ARMS) based on two independent sets of gene expression profiles: primary RMS tumors and RD ERMS cells transduced with inducible P3F constructs.

These paradoxical features of PAX3-FOXO1 being both oncogenic and growth-suppressive were demonstrated in previous studies with immortalized murine fibroblasts $(20,21)$ and human myoblasts (38). We identified and validated that tumor-suppressor genes, GREM1 (39) and DAPK1 (40), are upregulated at both RNA and protein levels in PAX3-FOXO1positive ARMS tumors and PAX3-FOXO1-ER inducible cell culture systems. Our study suggests that GREM1 and 
DAPK1 tumor suppressor genes can be potential target genes contributing to this growth-suppressive activity of high PAX3FOXO1 expression.

In conclusion, we identified 34 potential downstream target genes of PAX3(+/-Q)-FOXO1 by analyzing two independent sets of gene expression profiles: primary RMS tumors and RD ERMS cells transduced with inducible PAX3-FOXO1 constructs. Our study can serve as a basis to propose the 4 genes (GREM1,DAPK1, MYODI and HEYl) as targets that function in growth suppression or myogenic differentiation downstream of PAX3-FOXO1 in ARMS (Fig. 6).

\section{Acknowledgements}

This research was supported by NIH grants CA064202-13 (F.G. Barr), CA104896-03 (F.G. Barr), CA087812-06 (F.G. Barr, G.E. Mercado), and CA106450 (M. Ladanyi). We thank Dr Frederic G. Barr for his support and advice; Donna M. Gustafson for technical assistance; Dr Shujuan J. Xia for technical advice; Juseong Lee for editorial assistance; and Dr Lawrence A. Loeb (CA077852-14) for encouragement and comment.

\section{References}

1. Linardic CM: PAX3-FOXO1 fusion gene in rhabdomyosarcoma. Cancer Lett 270: 10-18, 2008.

2. Sorensen PH, Lynch JC, Qualman SJ, et al: PAX3-FKHR and PAX7-FKHR gene fusions are prognostic indicators in alveolar rhabdomyosarcoma: a report from the Children's Oncology Group. J Clin Oncol 20: 2672-2679, 2002.

3. Stevens MC: Treatment for childhood rhabdomyosarcoma: the cost of cure. Lancet Oncol 6: 77-84, 2005.

4. Scrable HJ, Witte DP, Lampkin BC and Cavenee WK: Chromosomal localization of the human rhabdomyosarcoma locus by mitotic recombination mapping. Nature 329: 645-647, 1987.

5. Visser M, Sijmons C, Bras J, et al: Allelotype of pediatric rhabdomyosarcoma. Oncogene 15: 1309-1314, 1997.

6. Davis RJ and Barr FG: Fusion genes resulting from alternative chromosomal translocations are overexpressed by gene-specific mechanisms in alveolar rhabdomyosarcoma. Proc Natl Acad Sci USA 94: 8047-8051, 1997.

7. Du S, Lawrence EJ, Strzelecki D, Rajput P, Xia SJ, Gottesman DM and Barr FG: Co-expression of alternatively spliced forms of PAX3, PAX7, PAX3-FKHR and PAX7-FKHR with distinct DNA binding and transactivation properties in rhabdomyosarcoma. Int J Cancer 115: 85-92, 2005.

8. Kelly KM, Womer RB, Sorensen PHB, Xiong QB and Barr FG: Common and variant gene fusions predict distinct clinical phenotypes in rhabdomyosarcoma. J Clin Oncol 15: 1831-1836, 1997.

9. Anderson J, Gordon T, McManus A, et al: Detection of the PAX3-FKHR fusion gene in paediatric rhabdomyosarcoma: a reproducible predictor of outcome? Br J Cancer 85: 831-835, 2001.

10. Khan J, Bittner ML, Saal LH, et al: cDNA microarrays detect activation of a myogenic transcription program by the PAX3-FKHR fusion oncogene. Proc Natl Acad Sci USA 96: 13264-13269, 1999.

11. Wachtel M, Dettling M, Koscielniak E, et al: Gene expression signatures identify rhabdomyosarcoma subtypes and detect a novel $\mathrm{t}(2 ; 2)(\mathrm{q} 35 ; \mathrm{q} 23)$ translocation fusing PAX3 to NCOA1. Cancer Res 64: 5539-5545, 2004.

12. Wachtel M, Runge T, Leuschner I, et al: Subtype and prognostic classification of rhabdomyosarcoma by immunohistochemistry. $\mathrm{J}$ Clin Oncol 24: 816-822, 2006.

13. Davicioni E, Finckenstein FG, Shahbazian V, Buckley JD, Triche TJ and Anderson MJ: Identification of a PAX-FKHR gene expression signature that defines molecular classes and determines the prognosis of alveolar rhabdomyosarcoma. Cancer Res 66: 6936-6946, 2006.
14. De Pittà C, Tombolan L, Albiero G, et al: Gene expression profiling identifies potential relevant genes in alveolar rhabdomyosarcoma pathogenesis and discriminates PAX3-FKHR positive and negative tumors. Int J Cancer 118: 2772-2781, 2006.

15. Laé M, Ahn EH, Mercado GE, et al: Global gene expression profiling of PAX-FKHR fusion-positive alveolar and PAX-FOXO1 fusion-negative embryonal rhabdomyosarcomas. J Pathol 212: 143-151, 2007.

16. Mercado GE, Xia SJ, Zhang C, et al: Identification of PAX3FKHR-regulated genes differentially expressed between alveolar and embryonal rhabdomyosarcoma: focus on MYCN as a biologically relevant target. Genes Chromosomes Cancer 47: 510-520, 2008.

17. Barr FG, Smith LM, Lynch JC, Strzelecki D, Parham DM, Qualman SJ and Breitfeld PP: Examination of gene fusion status in archival samples of alveolar rhabdomyosarcoma entered on the Intergroup Rhabdomyosarcoma Study-III trial: a report from the Children's Oncology Group. J Mol Diagn 8: 202-208, 2006.

18. Bennicelli JL, Edwards RH and Barr FG: Mechanism for transcriptional gain of function resulting from chromosomal translocation in alveolar rhabdomyosarcoma. Proc Natl Acad Sci USA 93: 5455-5459, 1996.

19. Tomescu O, Xia SJ, Strezlecki D, Bennicelli JL, Ginsberg J, Pawel B and Barr FG: Inducible short-term and stable long-term cell culture systems reveal that the PAX3-FKHR fusion oncoprotein regulates CXCR4, PAX3, and PAX7 expression. Lab Invest 84: 1060-1070, 2004.

20. Xia SJ and Barr FG: Analysis of the transforming and growth suppressive activities of the PAX3-FKHR oncoprotein. Oncogene 23: 6864-6871, 2004.

21. Xia SJ, Rajput P, Strzelecki DM and Barr FG: Analysis of genetic events that modulate the oncogenic and growth suppressive activities of the PAX3-FKHR fusion oncoprotein. Lab Invest 87: 318-325, 2007.

22. Littlewood TD, Hancock DC, Danielian PS, Parker MG and Evan GI: A modified oestrogen receptor ligand-binding domain as an improved switch for the regulation of heterologous proteins. Nucleic Acids Res 23: 1686-1690, 1995.

23. Tusher VG, Tibshirani R and Chu G: Significance analysis of microarrays applied to the ionizing radiation response. Proc Natl Acad Sci USA 98: 5116-5121, 2001.

24. Huang da W, Sherman BT and Lempicki RA: Systematic and integrative analysis of large gene lists using DAVID bioinformatics resources. Nat Protoc 4: 44-57, 2009.

25. Ahn EH and Schroeder JJ: Sphinganine causes early activation of JNK and p38 MAPK and inhibition of AKT in HT-29 human colon cancer cells. Anticancer Res 26: 121-127, 2006.

26. Tonin PN, Scrable H, Shimada H and Cavenee WK: Musclespecific gene expression in rhabdomyosarcomas and stages of human fetal skeletal muscle development. Cancer Res 51: 5100-5106, 1991.

27. Tapscott SJ, Thayer MJ and Weintraub H: Deficiency in rhabdomyosarcomas of a factor required for MyoD activity and myogenesis. Science 259: 1450-1453, 1993.

28. Ren YX, Finckenstein FG, Abdueva DA, et al: Mouse mesenchymal stem cells expressing PAX-FKHR form alveolar rhabdomyosarcomas by cooperating with secondary mutations. Cancer Res 68: 6587-6597, 2008.

29. Graf Finckenstein F, Shahbazian V, Davicioni E, Ren YX and Anderson MJ: PAX-FKHR function as pangenes by simultaneously inducing and inhibiting myogenesis. Oncogene 27: 2004-2014, 2008.

30. Sun J, Kamei CN, Layne MD, Jain MK, Liao JK, Lee ME and Chin MT: Regulation of myogenic terminal differentiation by the hairy-related transcription factor CHF2. J Biol Chem 276: 18591-18596, 2001.

31. Epstein JA, Lam P, Jepeal L, Maas RL and Shapiro DN: Pax3 inhibits myogenic differentiation of cultured myoblast cells. J Biol Chem 270: 11719-11722, 1995.

32. Ebauer M, Wachtel M, Niggli FK and Schäfer BW: Comparative expression profiling identifies an in vivo target gene signature with TFAP2B as a mediator of the survival function of PAX3/FKHR. Oncogene 26: 7267-7281, 2007.

33. Kikuchi K, Tsuchiya K, Otabe O, et al: Effects of PAX3-FKHR on malignant phenotypes in alveolar rhabdomyosarcoma. Biochem Biophys Res Commun 365: 568-574, 2008.

34. Collins MH, Zhao H, Womer RB and Barr FG: Proliferative and apoptotic differences between alveolar rhabdomyosarcoma subtypes: a comparative study of tumors containing PAX3-FKHR or PAX7-FKHR gene fusions. Med Pediatr Oncol 37: 83-89, 2001. 
35. Anderson J, Ramsay A, Gould S and Pritchard-Jones K: PAX3-FKHR induces morphological change and enhances cellular proliferation and invasion in rhabdomyosarcoma. Am J Pathol 159: 1089-1096, 2001.

36. Bernasconi M, Remppis A, Fredericks WJ, Rauscher FJ III and Schäfer BW: Induction of apoptosis in rhabdomyosarcoma cells through down-regulation of PAX proteins. Proc Natl Acad Sci USA 93: 13164-13169, 1996.

37. Ayyanathan K, Fredericks WJ, Berking C, Herlyn M, Balakrishnan C, Gunther E and Rauscher FJ III: Hormonedependent tumor regression in vivo by an inducible transcriptional repressor directed at the PAX3-FKHR oncogene. Cancer Res 60: 5803-5814, 2000.
38. Xia SJ, Holder DD, Pawel BR, Zhang C and Barr FG: High expression of the PAX3-FKHR oncoprotein is required to promote tumorigenesis of human myoblasts. Am J Pathol 175: 2600-2608, 2009.

39. Chen B, Athanasiou M, Gu Q and Blair DG: Drm/Gremlin transcriptionally activates p21(Cip1) via a novel mechanism and inhibits neoplastic transformation. Biochem Biophys Res Commun 295: 1135-1141, 2002.

40. Bialik S and Kimchi A: DAP-kinase as a target for drug design in cancer and diseases associated with accelerated cell death. Semin Cancer Biol 14: 283-294, 2004. 